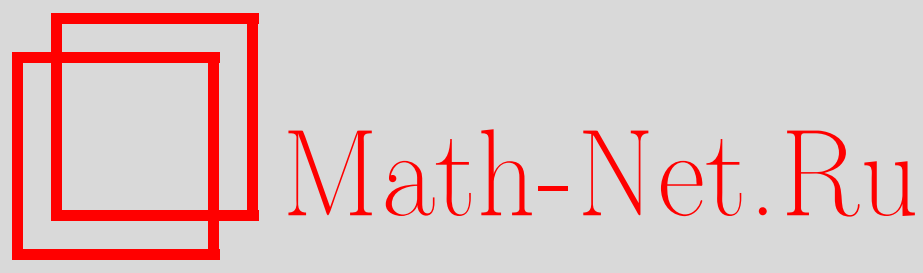

П. Л. Рубин, Дифференциальное уравнение для функционального интеграла, ТМФ, 2008, том 156, номep 2, 184-188

DOI: https://doi.org/10.4213/tmf6239

Использование Общероссийского математического портала Math-Net.Ru подразумевает, что вы прочитали и согласны с пользовательским соглашением http://www . mathnet.ru/rus/agreement

Параметры загрузки:

IP: 54.89 .56 .158

26 апреля 2023 г., 17:03:05

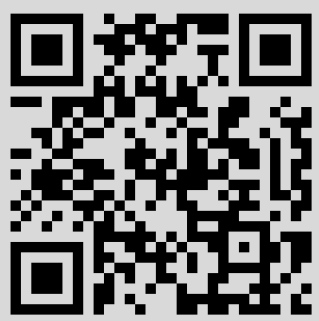




\title{
ДИФФЕРЕНЦИАЛЬНОЕ УРАВНЕНИЕ ДЛЯ ФУНКЦИОНАЛЬНОГО ИНТЕГРАЛА
}

\begin{abstract}
Предложен новый подход к вычислению функциональных интегралов в тех случаях, когда функционал, подлежащий усреднению (интегрированию), зависит от функций более чем одной переменной. Метод аналогичен тому, который использовал Фейнман в одномерном случае (квантовая механика). Рассмотрено интегрирование функционалов, зависящих от функций двух переменных и обладающих симметрией относительно поворотов вокруг некоторой точки плоскости. Предполагается, что функциональный интеграл берется по функциям, заданным в конечной области пространства (в круге радиуса $r$ ). Получено дифференциальное уравнение, описывающее изменение функционала по мере возрастания радиуса $r$.
\end{abstract}

Ключевые слова: функциональный интеграл, граничные условия.

\section{1. ВВЕДЕНИЕ}

Концепция интегрирования, согласно которой в роли переменной интегрирования выступает функция из некоторого функционального пространства, а объектом интегрирования является функционал, зависящий от этой функции, имеет уже достаточно долгую историю. Упомянем работу Орнштейна и Цернике [1] (хотя в ней явно понятие функционального интеграла еще не вводится), винеровский функциональный интеграл [2], работы Фейнмана, применившего функциональный интеграл в квантовой механике и квантовой теории поля [3]. В одномерном случае, когда функционал зависит от функции одной переменной, вычисление соответствующего функционального интеграла сводится к решению дифференциального уравнения в частных производных. В квантовой механике - это уравнение Шредингера.

В многомерном случае, когда функционал зависит от функции нескольких переменных, использовался другой метод вычисления интеграла. Он основан на том, что в случае свободных полей энергия системы является квадратичным функционалом переменных поля. При этом интеграл вычисляется достаточно просто. Аналогичная ситуация возникает и в статистической физике, когда энергию флуктуаций можно считать квадратичным функционалом флуктуирующих полей [4]. В тех случаях,

* Физический институт им. П. Н. Лебедева РАН, Москва, Россия.

E-mail: rubin@sci.lebedev.ru 
когда квадратичное приближение недостаточно (взаимодействующие поля, окрестность точки фазового перехода второго рода), использовался ряд теории возмущений, члены которого могут быть наглядно представлены с помощью фейнмановских диаграмм [4].

В настоящей работе рассматривается интеграл по функциям двух переменных. Разумеется, трехмерное и четырехмерное пространства важнее для приложений. Однако новый подход к задаче удобнее сначала рассмотреть в более простой ситуации. Этот подход применим к ограниченному, но все же довольно широкому классу функционалов, инвариантных относительно поворотов вокруг некоторой точки пространства. К их числу относится, в частности, корреляционная функция тепловых флуктуаций некоторого скалярного поля, характеризующего изотропную среду при отсутствии внешних полей. Для интеграла выводится дифференциальное уравнение в частных производных. Метод вывода уравнения в принципе близок к методу, использованному Фейнманом в квантовой механике [3], где функции, являющиеся "переменными интегрирования", заданы на конечном интервале времени и их значения на границах этого интервала фиксированы. Однако увеличение размерности пространства, на котором заданы эти функции, делает вывод более сложным.

\section{2. ПОСТАНОВКА ЗАДАЧИ}

Функциональный интеграл, который в первую очередь будет рассмотрен в настоящей работе, возник в задаче о критических флуктуациях. Пусть $f$ - скалярная величина, характеризующая некоторое локальное свойство рассматриваемого вещества. Предполагается, что $\langle f\rangle=0$. Угловые скобки означают термодинамическое усреднение, которое сводится к функциональному интегрированию. Если среда однородна, изотропна и не подвержена действию внешних полей, то корреляционная функция $C\left(x_{1}, x_{2}\right)=\left\langle f\left(x_{1}\right) f\left(x_{2}\right)\right\rangle$ инвариантна относительно вращений вокруг точек $x_{1}$ или $x_{2}$, хотя усредняемое выражение подобной симметрией не обладает. Однако результат интегрирования не изменится, если предварительно усреднению подвергнуть исходный функционал, т.е. принять, что

$$
C\left(x_{1}, x_{2}\right)=\left\langle\frac{1}{2 \pi} \oint f(0) f(R, \varphi) d \varphi\right\rangle .
$$

Начало системы полярных координат может быть помещено в одну из двух рассматриваемых точек (для определенности - в точку $\left.x_{1}\right)$, тогда $(R, \varphi)$ - координаты точки $x_{2}, R=\left|x_{1}-x_{2}\right|$. Теперь уже не только результат интегрирования, но и само интегрируемое выражение инвариантны относительно поворотов вокруг начала координат.

Корреляционную функцию можно записать следующим образом (см. [5], § 147):

$$
C\left(x_{1}, x_{2}\right)=\int f\left(x_{1}\right) f\left(x_{2}\right) e^{-E(f)} D f=\int\left(\frac{1}{2 \pi} \oint f(0) f(R, \varphi) d \varphi\right) e^{-E(f)} D f .
$$

Функционал $E(f)$ имеет вид

$$
E(f)=\int\left(\frac{a}{2}(\nabla f(x))^{2}+U[f(x)]\right) d x
$$


где $a$ - постоянная величина, а функция $U[f]$ предполагается ограниченной снизу, четной и достаточно быстро возрастающей при увеличении $|f|$. В задаче о критических флуктуациях в изотропной среде и при отсутствии внешнего поля обычно полагают (см. [5], § 147)

$$
U[f]=b f^{2}+g f^{4},
$$

где $b$ и $g-$ постоянные величины, $g>0^{1)}$.

Теперь постановка задачи будет несколько изменена в связи с предлагаемым способом вычисления интеграла. Пусть функции, по которым производится интегрирование (усреднение), имеют ограниченный носитель и сосредоточены внутри круга радиуса $r$ (круг обозначим $K_{r}$, а ограничивающую его окружность $-O_{r}$ ). В задаче о флуктуациях такая ситуация возникает, если образец ограничен и занимает круг $K_{r}$. Начало координат по-прежнему находится в точке $x_{1}$. Будем считать, что там же находится и центр круга $K_{r}$. Координаты точки обозначим $\rho$ и $\varphi$ (окружность $\rho=r$ - это граница $K_{r}$ ). Нормировка интеграла определяется так, чтобы среднее значение константы равнялось самой константе: $\langle 1\rangle_{K_{r}}=1$. Символ $\langle\ldots\rangle_{K_{r}}$ означает усреднение, выполненное по функциям с носителем внутри $K_{r}$. Аналогичные обозначения будут применяться и в дальнейшем: нижний индекс у правой угловой скобки будет обозначать область, где сосредоточены носители функций, по которым выполняется усреднение (функциональное интегрирование).

Как и в одномерном случае (см. [3]) будем считать, что значение функции $f(\rho, \varphi)$, играющей роль переменной интегрирования, фиксировано на границе круга $O_{r}$ :

$$
f(r, \varphi)=u(\varphi)
$$

где $u(\varphi)$ - некоторая заданная функция. Функциональный интеграл также обозначим $C$, но аргументы $C$ изменятся (теперь в общем случае $C$ представляет собой функционал):

$$
C(R, r, u)=\int\left(\frac{1}{2 \pi} \oint f(0) f(R, \varphi) d \varphi\right) e^{-E(f)} \hat{\delta}[r, f(r, \varphi)-u(\varphi)] D f,
$$

где $\hat{\delta}[r, f(r, \varphi)-u(\varphi)]$ - функциональный аналог $\delta$-функции, означающий, что на окружности $O_{r}$ функция $f(r, \varphi)=u(\varphi)$. Радиус круга будет считаться переменным, и корреляционная функция $C\left(x_{1}, x_{2}\right)$ (см. (1)) должна получиться при переходе в равенстве $(3)$ к пределу при $r \rightarrow \infty$. При этом результат не должен зависеть от величины $u$ на границе круга $K_{r}$ (см. сноску 1$)$.

\section{3. ЭВОЛЮЦИОННОЕ УРАВНЕНИЕ}

Дальнейшие рассуждения в значительной мере повторяют использованный Фейнманом [3] вывод уравнения, но с учетом изменения размерности пространства. Цель этих рассуждений - получить уравнение (7), описывающее изменение некоторого функционала $\phi(r, u)$ по мере возрастания радиуса $r$. При увеличении радиуса круга

\footnotetext{
1) Ситуация после достижения точки фазового перехода, т.е. сосуществование двух фаз, здесь не рассматривается. Но сколь угодно близкий подход к точке фазового перехода допускается.
} 
от $r$ до $r+\varepsilon$ функция в кольце между кругами указанных радиусов записывается в виде (ср. [3])

$$
f(\rho, \varphi)=\frac{\rho-r}{\varepsilon} w(\varphi)+u(\varphi)
$$

где величина $u(\varphi)$ фиксирована, а по $\rho$ и $w(\varphi)$ надо выполнить интегрирование. Упомянутое кольцо обозначим $\delta K$ (радиусы кругов, ограничивающих кольцо, в этом обозначении явно не указываются).

Рассмотрим приращение функционала

$$
\begin{aligned}
\Delta C & =C(R, r+\varepsilon, u)-C(R, r, u)= \\
& =\oint \frac{\delta C(R, r, u)}{\delta u(\varphi)}\langle w(\varphi)\rangle_{\delta K} d \varphi+\frac{1}{2} \oint \frac{\delta^{2} C(R, r, u)}{\delta u\left(\varphi_{1}\right) \delta u\left(\varphi_{2}\right)}\left\langle w\left(\varphi_{1}\right) w\left(\varphi_{2}\right)\right\rangle_{\delta K} d \varphi_{1} d \varphi_{2}+\cdots .
\end{aligned}
$$

Первое слагаемое в правой части этого равенства обращается в нуль вследствие предполагаемой четности $U[f]$ (см. выше), так что

$$
\begin{aligned}
\Delta C & =C(R, r+\varepsilon, u)-C(R, r, u)= \\
& =\frac{1}{2} \oint \frac{\delta^{2} C(R, r, u)}{\delta u\left(\varphi_{1}\right) \delta u\left(\varphi_{2}\right)}\left\langle w\left(\varphi_{1}\right) w\left(\varphi_{2}\right)\right\rangle_{\delta K} d \varphi_{1} d \varphi_{2}+\cdots .
\end{aligned}
$$

Из (1) и (2) следует, что при $\varepsilon \rightarrow 0$ в кольце $\delta K$ основной вклад в интеграл вносят только малые значения $w,|w| \rightarrow 0$. Поэтому с точностью до членов 1-го порядка по $\varepsilon$ величину $U[f]$ можно заменить на $U[u]$. После такой замены интеграл по $w$ становится гауссовым. Вычислим этот интеграл, заменяя $\varphi$ на новую переменную $l=\rho \varphi$ и полагая $w(\varphi)=v(l)$.

Поскольку теперь величину $U[f]$ при интегрировании по $f$ можно считать постоянной, функционал $E(f)($ см. $(2))$ при интегрировании по кольцу $\delta K$ можно записать следующим образом:

$$
\int_{\delta K} \frac{a}{2}(\nabla f)^{2} d \rho d l=\frac{a}{2} \oint d l\left(\frac{v(l)^{2}}{\varepsilon}-\frac{\varepsilon}{3} v(l) v^{\prime \prime}(l)\right) .
$$

В правой части этого равенства выполнено интегрирование по частям с учетом того, что область интегрирования (окружность) замкнута. Величина $\left\langle w\left(\varphi_{1}\right) w\left(\varphi_{2}\right)\right\rangle_{\delta K}$ вычисляется стандартным образом (см. [4]):

$$
\left\langle w\left(\varphi_{1}\right) w\left(\varphi_{2}\right)\right\rangle_{\delta K}=\left\langle v\left(l_{1}\right) v\left(l_{2}\right)\right\rangle_{\delta K}=\frac{\sqrt{3}}{a} \exp \left[-\frac{\sqrt{3}\left|l_{1}-l_{2}\right|}{\varepsilon}\right] .
$$

Теперь уже с помощью (4) несложно получить эволюционное уравнение для $C(R, r, u)$

$$
\frac{\partial C(R, r, u)}{\partial r}=\frac{1}{2 r} \oint \frac{\delta^{2} C(R, r, u)}{\delta u(\varphi)^{2}} d \varphi-r C(R, r, u) \oint U[u(\varphi)] d \varphi .
$$

\section{4. ДИФФЕРЕНЦИАЛЬНОЕ УРАВНЕНИЕ}

Цель дальнейших рассуждений - упростить решаемую задачу. Будем считать "граничные" функции $u$ постоянными. Тогда $C(R, r, u)$ становится не функционалом, а функцией. Рассмотрим связь между производными функционала и функции, 
получающейся из него в результате сделанного упрощения. Функционал $n$-й степени имеет вид

$$
\phi_{n}(u)=\underbrace{\oint \ldots \oint}_{n} G\left(\varphi_{1}, \ldots, \varphi_{n}\right) u\left(\varphi_{1}\right) \ldots u\left(\varphi_{n}\right) d \varphi_{1} \ldots d \varphi_{n} .
$$

Если $G\left(\varphi_{1}, \ldots, \varphi_{n}\right)=$ const, то этот функционал инвариантен относительно вращений системы координат на любой угол

$$
\phi_{n}(u)=G(\oint u(\varphi) d \varphi)^{n} .
$$

Отметим, что формула (6) дает не самый общий вид функционала, инвариантного при вращениях. Но если он является таким при некотором $r$, то согласно (5), он останется таким же и при возрастании $r^{2)}$.

Пусть $F_{n}(u)$ - функция, которая получается из этого функционала при замене функции $u(\varphi)$ на постоянную величину $u, F_{n}(u)=G(2 \pi u)^{n}$, при этом

$$
\left.\oint d \varphi \frac{\delta^{2} \phi_{n}(u)}{\delta u(\varphi)^{2}}\right|_{u=\mathrm{const}}=\frac{1}{2 \pi} \frac{\partial^{2} F_{n}(u)}{\partial u^{2}} .
$$

Поскольку это равенство справедливо при любом $n$, оно должно быть верным и в общем случае:

и, в частности, для

$$
\left.\oint d \varphi \frac{\delta^{2} \phi(u)}{\delta u(\varphi)^{2}}\right|_{u=\mathrm{const}}=\frac{1}{2 \pi} \frac{\partial^{2} F(u)}{\partial u^{2}},
$$

$$
F(R, r, u)=\left.C(R, r, u)\right|_{u=\text { const }} .
$$

С учетом сказанного выше уравнение (5) для рассматриваемого функционала принимает вид

$$
\frac{\partial F(r, u)}{\partial r}-\frac{1}{4 \pi r} \frac{\partial^{2} F(r, u)}{\partial u^{2}}+2 \pi r U(u) F(r, u)=0 .
$$

Вывод этого “эволюционного” по $r$ уравнения и являлся целью настоящей работы.

Благодарности. Автор благодарит Р. А. Минлоса, С. А. Пирогова и И. В. Тютина за обсуждение работы.

\section{Список литературы}

[1] L.S. Ornstein, F. Zernike, Proc. Kon. Akad. Wet., 17 (1914), 793.

[2] М. Кац, Вероятность и смежные вопросы в физике, Мир, М., 1965.

[3] Р. Фейнман, А. Хибс, Квантовая механика и интегралы по траекториям, Мир, М., 1968.

[4] В.Н. Попов, Континуальные интегралы в квантовой теории поля и статистической физике, Атомиздат, М., 1976.

[5] Л. Д. Ландау, Е. М. Лифшиц, Теоретическая физика. Т. 5: Статистическая физика. Часть 1, Физматлит, М., 2001.

Поступила в редакцию 8.05.2007, после доработки 8.10.2007

\footnotetext{
2)Уравнение (5) остается справедливым для любого функционала вида (3), если усредняемый функционал зависит только от значений функций $f$ в круге $K_{r}$.
} 